\title{
A novel, adaptable, radiographically opaque, multi-plane continuous filament marker for optimizing tissue identification, radiation planning, and radiographic follow-up
}

\author{
Sunny Mitchell ${ }^{1}$, Henry Lee ${ }^{2}$, Beth Baughman DuPree ${ }^{3}$, David C. Beyer ${ }^{4}$, Michael Ulissey ${ }^{5}$, \\ Stephen R. Grobmyer ${ }^{6}$, Jennifer Gass ${ }^{7}$, Susan Boolbol ${ }^{8}$, Toni Storm-Dickerson ${ }^{9}$
}

${ }^{1}$ Department of Surgery, Montefiore Nyack Hospital, Nyack, NY, USA; ${ }^{2}$ Department of Radiation Oncology; New York Presbyterian-Lawrence Hospital, Columbia University College of Physicians \& Surgeons, Bronxville, NY, USA; ${ }^{3}$ Sedona Breast care, Department of Surgery, Cancer Centers of Northern Arizona Healthcare, Sedona, AZ, USA; ${ }^{4}$ Department of Radiation Oncology, Cancer Centers of Northern Arizona Healthcare, Sedona, AZ, USA; ${ }^{5}$ Department of Radiology, The University of Texas Health Sciences Center, San Antonio, TX, USA; ${ }^{6}$ Oncology Institute, Cleveland Clinic Abu Dhabi, Abu Dhabi, United Arab Emirates; ${ }^{7}$ Department of Surgery, Women \& Infants Hospital, Brown University, Providence, RI, USA; ${ }^{8}$ Department of Surgery, Mount Sinai Beth Israel, Icahn School of Medicine, New York, NY, USA; ${ }^{9}$ Department of Surgery, Kearney Breast Center, Vancouver, WA, USA

Contributions: (I) Conception and design: All authors; (II) Administrative support: None; (III) Provision of study materials or patients: All authors; (IV) Collection and assembly of data: All authors; (V) Data analysis and interpretation: All authors; (VI) Manuscript writing: All authors; (VII) Final approval of manuscript: All authors.

Correspondence to: Sunny Mitchell, MD. Department of Surgery, Montefiore Nyack Hospital, 160 N. Midland Ave, Nyack, NY 10960, USA.

Email: mitchellsun@yahoo.com.

Background: In breast cancer treatment, marking the tumor bed is an important aspect of the surgical component of therapy. Clear delineation of the tumor bed allows radiation oncologists a defined target for planning and delivering postoperative radiation therapy (XRT). Tumor bed marking also allows radiographic follow-up of the tumor bed on subsequent breast imaging. The aim of this assessment is to evaluate the ease and feasibility of utilizing a tumor bed filament marker (VeraForm ${ }^{\circledR}$, Videra Surgical inc., USA) as a marker in post-operative benign surgical sites and malignant breast surgical tumor beds in breast cancer surgery.

Methods: The filament marker is a novel radiopaque surgical filament that in lieu of clips and other markers is implanted in the surgical tumor bed during breast surgery. Following development of the filament marker, the researchers used breast phantoms and radiographic images to develop a series of geometric patterns of placement options that optimize comprehensive multi-plane radiographic interpretation of the exact tumor bed or surgical margin. Three breast surgeons at 3 separate institutions then used this filament as a continuous multi-plane marker in 20 patients during breast conservation surgery. In these patients, the filament marker was thus used to mark the tumor bed (breast cancer surgery) or surgical site (benign breast disease) instead of the more traditional devices such as clips or other metallic open framework devices. We then assessed 2 important factors related to this device; (I) the ease, feasibility, and accuracy of in vivo placement with oncoplastic and non-oncoplastic breast conservation surgery techniques; (II) the radiographic footprint this device left on standard imaging protocols of post-operative mammogram (MMG), computed tomography (CT) scan, breast magnetic resonance imaging (MRI) examinations, and ultrasounds (USs) for both routine follow-up imaging and for standard radiation planning.

Results: There were no adverse events reported with the use of this device. The cases were then reviewed by a multidisciplinary team that included the original surgeon, a breast radiologist, and radiation oncologist. Their unanimous evaluation was that the filament marker clearly delineated all sides and planes of the tumor bed (cancer surgery) or surgical site (benign disease). Regardless of surgical technique utilized, this information provided precise 3D guidance for radiation planning and delivery as well as radiographic followup. The surgeons involved reported that delineating the bed with the filament marker was a quick and easy procedure and did not interfere with performing the planned surgical technique. Radiologists, surgeons, 
and radiation oncologists found that the filament marker was not only radiographically opaque on CT and MMG, but also caused no significant artifact on CT, MRI, US, or MMG.

Conclusions: The continuous multi-plane filament marker is a new device that fulfills the heretofore unmet need for safe and improved tumor bed and tissue site marking. It is an easy to place, non-palpable continuous multi-plane radiographic opaque tissue marker that seems to better delineate the tumor bed, regardless of type of breast surgery performed, while providing a more accurate $3 \mathrm{D}$ image for radiation planning and radiographic follow-up on MMG MRI, CT and US.

Keywords: Breast cancer; breast surgery; oncoplastic; radiation planning; tumor bed marker; filament marker

Submitted Jun 12, 2019. Accepted for publication Sep 27, 2019.

doi: 10.21037 /gs.2019.10.03

View this article at: http://dx.doi.org/10.21037/gs.2019.10.03

\section{Introduction}

\section{Tumor bed marking rationale}

In the setting of breast conservation surgery (BCS), accurate and complete tumor bed identification for radiation planning and delivery to the tumor bed is an essential component of breast cancer treatment. Surgical site marking of an excisional biopsy and tumor bed marking of a lumpectomy site is utilized by radiologists, radiation therapists, and surgeons. Radiation treatment protocols entail identification of the tumor excision site or "tumor bed' within the breast surgical site in order to accurately deliver therapy. The tumor bed changes over time (1). As no prior method exists to accurately and reliably mark the entire tumor bed in all dimension (entire length of each margin: anterior, posterior, superior, inferior, medial and lateral), radiation oncologists are left to rely on surrogates as best possible guesstimates for tumor bed location, shape, and size of the tumor bed. This may result in an increase in delivered radiation therapy (XRT) beyond the actual tumor bed location (2-9), alteration of management of a boost (10-12), as well as a geographic miss on one or more margins. Unlike many other parts of the body, the breast lacks internal discernable anatomic landmarks to facilitate accurate targeted radiation delivery or targeted radiographic follow-up of a surgical tumor bed.

\section{Effect of breast surgical technique on tumor bed identification}

Over the last decade, the popularity of new surgical techniques utilized to accomplish BCS created a greater challenge for radiation oncologists (9) and radiologists to precisely identify margins. Potential surgical techniques range from a simple lumpectomy with superficial closure only; to simple lumpectomy with full thickness tumor bed closure directly under the overlying skin incision; to oncoplastic techniques involving an extended overlying skin incision with resulting scar (13-15) which may not necessarily represent the size/shape of the underlying tumor bed; to performing an oncoplastic BCS via a remote incision (16) leaving no overlying indication of where the tumor bed is; to utilizing a single incision and laparoscopic equipment to perform a quadrantectomy and axillary surgery (17); and to flap reconstruction in the setting of BCS which may move the tumor bed margins into the surrounding quadrant(s) (18). This variability translates into an inability to rely on the presence of a seroma, overlying scar, or cosmetic defect to predict where the tumor bed truly is.

\section{Traditionally utilized tumor bed markers}

Traditionally utilized tumor bed markers are associated with an inability to accurately identify the actual tumor bed margins (2-12) (superior, inferior, anterior, posterior, medial, lateral) in their entirety as well as potential artifact. As a result, the necessity of utilizing multiple surrogates in attempts to identify the most probable location of the tumor bed as well as measurements of shape and size of margins is created. Tumor bed surrogates used to extrapolate Breast tumor bed location and extent have included: seroma cavity, surgical clips, BioZorb ${ }^{\circledast}$ breast marker, fiducials, Operative Note, and pre-operative imaging. As described below, the inadequacy of these older tumor bed markers results in uncertainty for radiation oncologists and radiologists to accurately identify the tumor bed. 


\section{Seroma}

A post-operative seroma may be visible in any type of BCS but is more consistent in appearance when only performing a superficial closure. A full thickness closure of the tumor bed typically leads to diminution of or decreased potential for seroma formation (16). A seroma may represent the tumor bed, part of the tumor bed, the entire surgical field, or a combination of the above. An intrinsic characteristic of seromas is the potential variability of changing size and or shape over time $(9,19)$. Seroma and Surgical clip in the setting of non-Oncoplastic surgery (8) has also been utilized. Thus, due to the inconsistent presence, and lack of confidence that a seroma represents the true tumor bed, it is not a reliable or accurate marker.

\section{Surgical clips}

Surgical clips are the most common tumor bed markers in breast surgery. They are generally available in most ORs, but inconsistently utilized in breast surgery cases $(11,20)$. Despite being a standard in tumor bed marking, Yang et al. (9) report the accuracy of clips representing the original tumor site is debatable as they: may be displaced with full thickness closure of the surrounding tissue, thus not correlate with the true extent of the cavity; may not be easily visualized on portal images and only provide a single point of reference for a margin, leading observers to interpolate the border of the cavity $(2,12,21-28)$, potentially contributing to inaccuracy in target delineation for XRT. Radiation oncologists report alterations in delivered therapy secondary to clip placement $(11,24,27,29)$.

The exact recommended placement and number of surgical clips for tumor bed identification with radiation planning varies $(2,18,21-24,28,29)$. Clips have been reported to move or 'migrate' from their initial placement location (9,24,25,30-32). Clip migration was noted to be similar between groups of patients' XRT 100 days (median 53 days) from surgery to group starting afterward (median 163 days) (31). Clip migration after conventional breast surgery (closing the breast superficially) in comparison to lumpectomy with partial breast reconstruction techniques (sutured cavity) may not differ significantly (31). A potential relationship to body mass index and clip migration may has also been reported (30). Surgical techniques to decrease potential for displacement has been described (16).

Clips present on post-operative imaging may represent markers for tumor bed location or evidence of routine surgical techniques of hemostasis or lymphatic ligation. Thus, due to the inconsistent presence, limited identification of a single point along a tumor bed wall, inconsistent detectability on imaging, potential for movement/displacement and inability to delineate the extent of the tumor cavity (the full length of each margin wall), clips are not a reliable or accurate marker.

\section{Fiducials}

Fiducial markers have been cited as advantageous compared to bony landmarks for image guided partial breast irradiation set up accuracy (33), as well as superior to titanium clips placed at the surgeon's discretion when placed in each of the seroma cavity walls for visualization of the seroma cavity. Fiducials are not generally associated with radiographic artifact. Carbon fiducials can accurately represent the location of the cavity and may translate to a reduced whole breast irradiation (WBI) boost to approximately $4 \mathrm{~mm}$ (34). Gold fiducial markers are, however, another example of providing a single reference point along a tumor margin, resulting in the same debatable accuracy in representing the original tumor site as described by Yang et al. (9) in regards to surgical clips.

\section{BioZorb $^{\circledR}$}

A three-dimensional re-absorbable implantable device, BioZorb $^{\circledast}$, containing 6 permanent titanium clips, was designed to create a tumor bed target, resulting in a 'reduction of volume' for XRT delivery $(35,36)$ at the surgical bed, and act as a 'scaffold'/filler (36,37). To mitigate movement seen by traditional clips, an absorbable scaffold was created with integrated clips to inhibit any immediate movement. The space occupying component of the device is said to eventually reabsorb (37). Placement technique entails utilization of a sizer set to choose best fit for the marker (36) from a stock of markers, fitting the tumor bed to the marker size/shape available. A smaller marker, or avoidance of utilization of the marker is recommended if a paucity of overlying tissue exists (36). Biozorb ${ }^{\varpi}$ has not demonstrated value given its relative high cost and lack of clinical advantage scientifically over the use of surgical clips (38).

\section{Methods}

To meet the unfilled need of accurate tumor bed marking for radiation planning and delivery as well as radiographic follow-up, the researchers evaluated the ease and feasibility of utilization of the continuous multi-plane filament marker and its radiographic appearance on post-operative imaging during routine utilization by three multidisciplinary breast 


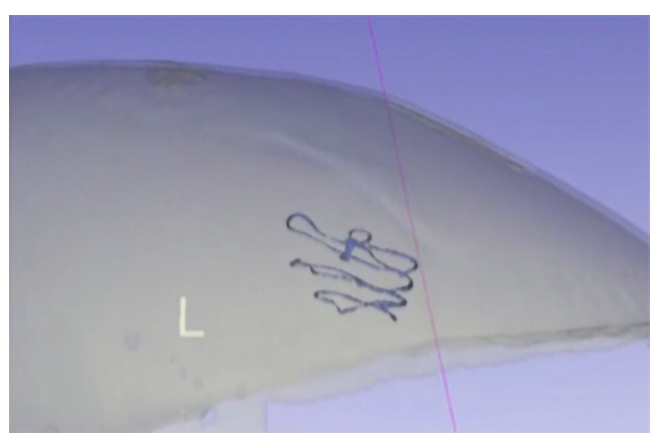

Figure 1 Visibility of lumpectomy tumor bed marked with the filament marker in breast phantom on CT XRT planning. CT, computed tomography; XRT, radiation therapy.

cancer treatment teams. The filament marker assessed is an FDA cleared non-palpable radiopaque continuous multi-plane marker that is threaded into the contour of the tumor cavity intra-operatively. It is inherently malleable to any tumor bed or surgical site, allowing the surgeon to adapt placement to the specific procedure performed, and simultaneously creating a $3 \mathrm{D}$ planning target for radiation oncologists. Proof of principle of complete tumor bed marking (anterior, posterior, superior, inferior, lateral, medial margins) and adaptability to surgical technique performed was initially demonstrated via placing the filament marker into breast phantoms and subsequent computed tomography (CT) (Figure 1).

Within the breast phantoms, the filament marker was utilized in the settings of oncoplastic tissue mobilization, simple lumpectomy with re-approximation of surrounding tissue, and in a simulated seroma. Multiple patterns were trialed to identify configurations that were easy, facile, and efficient to lay out by the surgeon with minimal time requirement. CT's for external beam XRT were obtained on the phantoms with the filament marker in place to assess ease and feasibility of rapid interpretation of the exact marked tumor bed within the surgical site and the ability to interpret all margins clearly (anterior, posterior, superior, inferior, medial, and lateral) without any discernable artifact (Figure 1). Once effective configurations were identified, ease and feasibility were further demonstrated in vivo via intraoperative placement during BCS and excisional biopsy surgeries with subsequent radiographic follow-up imaging on mammogram (MMG), ultrasound (US), magnetic resonance imaging (MRI), and CT planning for XRT using standard, commercially available systems. Three Breast Surgeons at 3 centers; Sedona Breast Center, Beth Israel
Mt. Sinai, and Women \& Infants Brown University Medical Center, performed routine tumor bed or surgical site marking with the filament marker in 20 patients undergoing 24 BCS. Patients were consented for the utilization of their de-identified medical information and images for this assessment of ease and feasibility of the filament marker utilization during routine breast surgery tumor bed and surgical site marking and subsequent radiographic followup and XRT CT planning. As this was an assessment of use and radiographic findings of utilization during routine BCS tumor bed and biopsy site marking, each surgeon performed BCS in their otherwise usual manner and used the type of BCS that is common to his or her practice. Furthermore, no limitations or restrictions were placed on the type and nature of preoperative care, incision site, dissection technique, resection of the lesion, surgical reconstruction, surgical closure, or post-operative care (tissue was assessed in the usual manner of each institution); and final surgical margins were pathologically assessed per institution standard. Routine post-operative imaging (MMG, XRT CT planning, US and MRI) were assessed for quality of radiographic opacity, related potential artifact, and complete (anterior, posterior, superior, inferior, lateral, and medial) margin delineation of the filament marker by the multidisciplinary breast team.

\section{Statement of ethics approval}

Patients gave informed consent for utilization of their deidentified images and medical information for the purposes of research and education. No ethics approval was required for the purposes of this reporting of findings. Patients were consented for the utilization of their de-identified medical information and images for this assessment of ease and feasibility of the filament marker utilization during routine breast surgery tumor bed and surgical site marking and subsequent radiographic follow-up and XRT CT planning. As this was an assessment of use and radiographic findings of utilization during routine BCS tumor bed and biopsy site marking, each surgeon performed BCS in their otherwise usual manner and used the type of BCS that is common to his or her practice. Furthermore, no limitations or restrictions were placed on the type and nature of preoperative care, incision site, dissection technique, resection of the lesion, surgical reconstruction, surgical closure, or post-operative care (tissue was assessed in the usual manner of each institution); and final surgical margins were pathologically assessed per institution standard. 

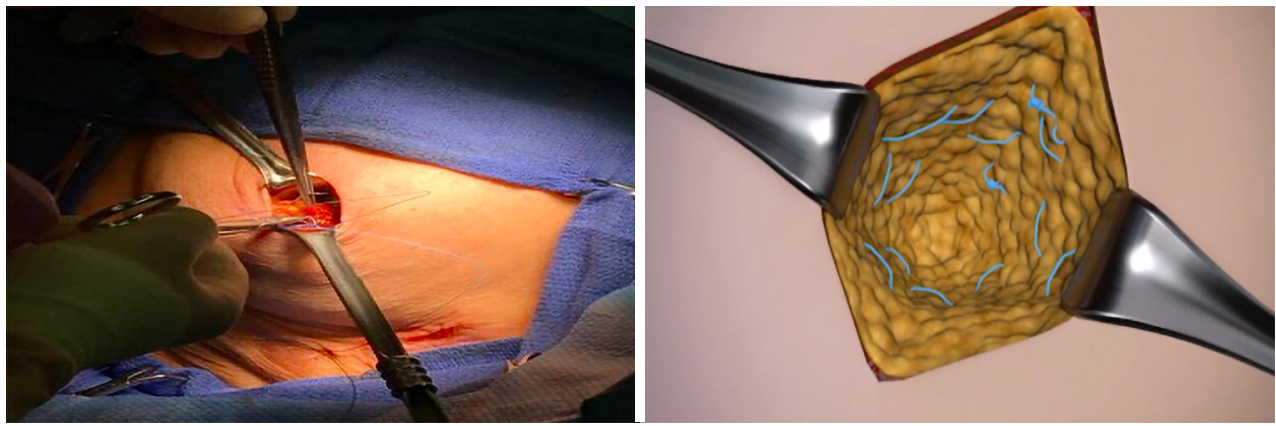

Figure 2 Intra-operative placement. (A) Intra-operative tumor bed marking with the filament marker utilizing a spiral configuration; (B) schemata of spiral configuration utilized intra-op with filament marker placement.
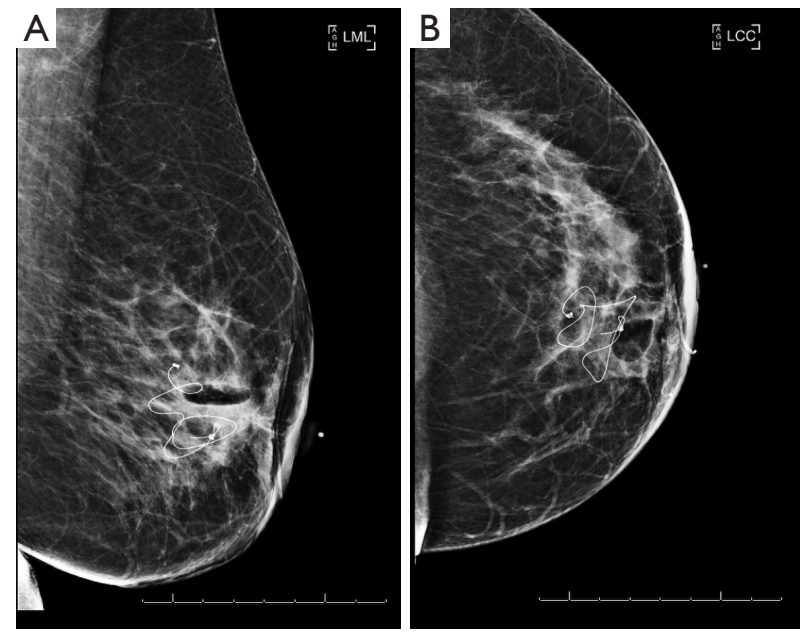

Figure 3 Four-week post-operative pre-XRT left breast mammogram of 61 years old female after left breast lumpectomy (+/+/- $1.2 \mathrm{~cm}$ invasive ductal carcinoma), tumor bed marked intra-operatively with the filament marker. XRT, radiation therapy.

Routine post-operative imaging (MMG, XRT CT planning, US and MRI) were assessed for quality of radiographic opacity, related potential artifact, and complete (anterior, posterior, superior, inferior, lateral, and medial) margin delineation of the filament marker by the multidisciplinary breast team.

\section{Results}

Three Breast Surgeons at the three aforementioned multidisciplinary Breast Centers performed routine tumor bed or surgical site marking with the filament marker (24 procedures in 20 patients) undergoing a lumpectomy (BCS) or excisional breast biopsy, (via simple full thickness closure,

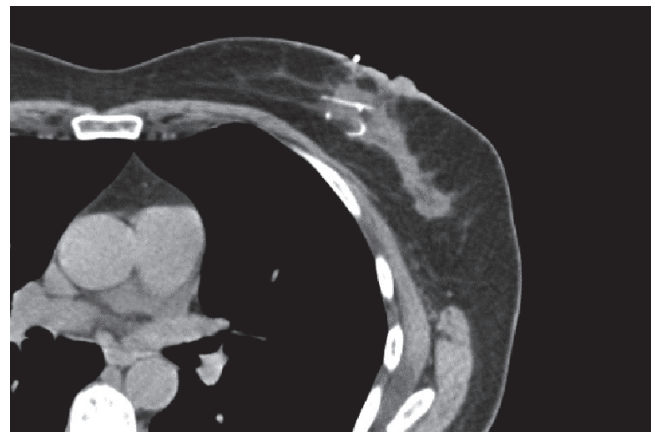

Figure 4 Five-week post-operative CT, left breast lumpectomy tumor bed marked with the filament marker. CT, computed tomography.

superficial closure, or oncoplastic technique with and without associated reduction mastopexy) from FebruaryMay 2019 (Figure 2).

The filament marker was placed in single site cases, bilateral cases, and multiple ipsilateral site cases. In two cases with post-operative positive margins, the filament marker was utilized to identify the exact tumor bed margins, removed with margin re-excision and replaced at the new margins. The surgeons reported the following: deployment was facile and took approximately 4 min per tumor bed marking on average; ease with placement regardless of surgical technique; and no interference with chosen tumor bed or surgical site closure. Surgeons and patients reported a consistent non-palpable nature. Patients reported no complaints. No patients experienced an infection and no patients required removal of the filament marker. Radiographic follow-up images portrayed an easily visible, continuous multiplane marker with negligible to no artifact on MMG (Figure 3), CT (Figure 4), and CT planning XRT 

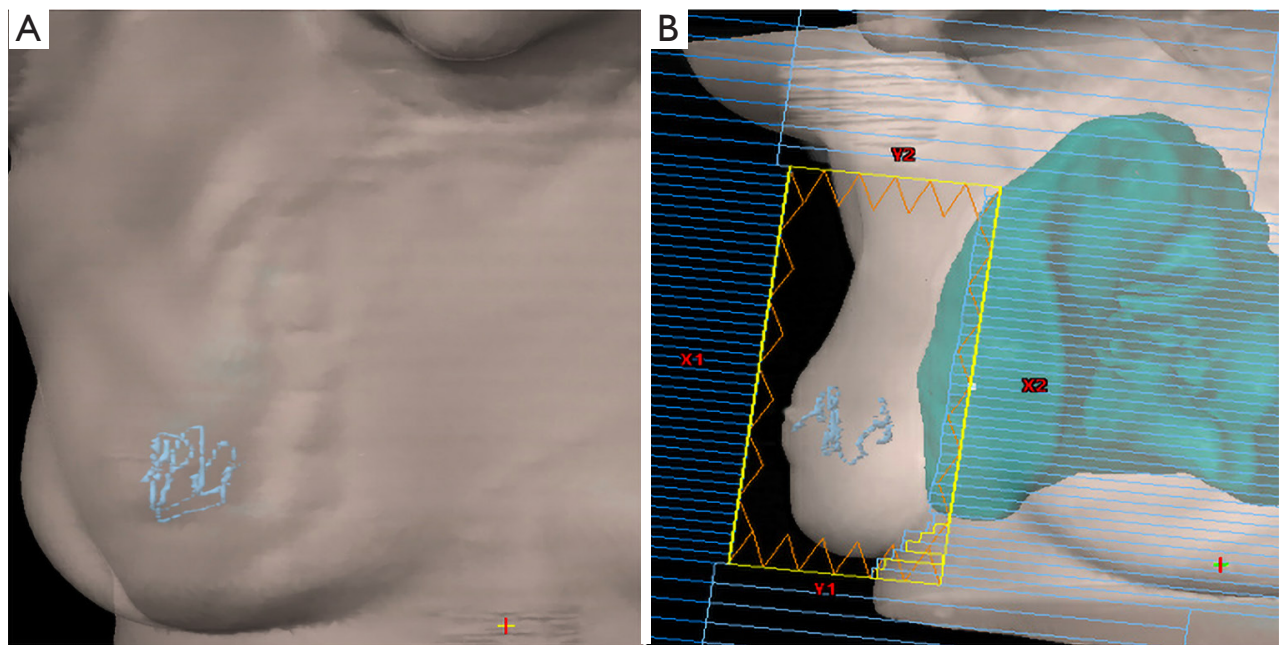

Figure 5 Tracing of the filament marker is reconstructed based on CT images and seen through the translucent skin rendering. (A) the anterior view and (B) a beams eye view with the filament marker, breast contour and projection of the lung identified. CT, computed tomography.

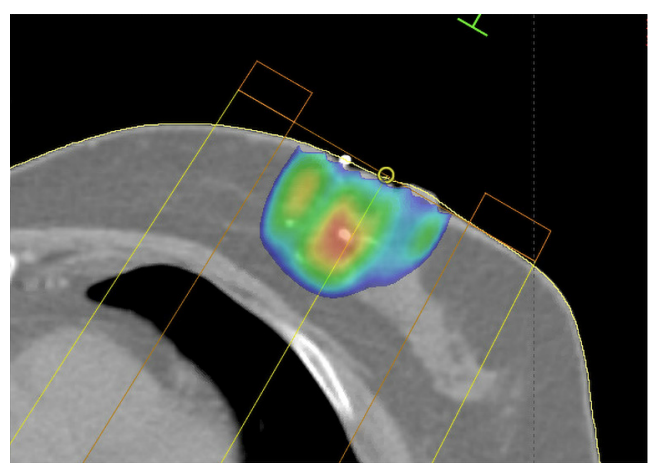

Figure 6 Five-week post-operative XRT planning CT with the filament marker. The color wash defines the intensity of dose throughout the region. Redder for higher dose and bluer for lower dose, but the area shown is all receiving at least the prescription dose. The marker is clearly visible within the region and the absence of significant radiation in the rest of the breast is also evident. This is the same patient as in Figure 4. CT, computed tomography; XRT, radiation therapy.

planning (Figures 5,6). The filament marker was either negligibly or not visible on US (Figure 7) and MRI (Figure 8) with negligible to no artifact. The exact site and extent of the tumor bed was consistently easily discernable from surrounding or nearby post-operative surgical changes (Figures 3-6,9).

No issues were noted with Mammotome ${ }^{\circledR}$ utilization when the filament marker was in place, the device was noted

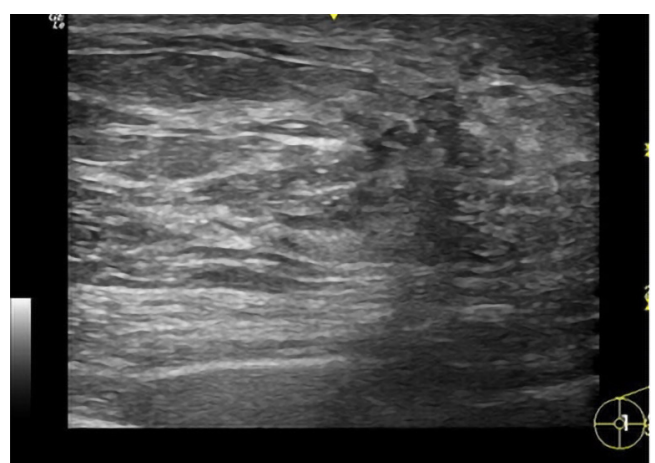

Figure 7 Post-operative ultrasound of lumpectomy site, tumor bed marked with the filament marker.

to cut right through the filament marker without incident. The majority of lumpectomies, oncoplastic or nononcoplastic, were amenable to a spiral configuration. Larger full thickness oncoplastic procedures without contiguous walls were more amenable to individual wall marking. Excisional biopsies may best be served with a simple stitch. The learning curve was minimal as surgeons routinely utilize sutures, needle drivers, and scissors.

\section{Discussion}

The ideal tumor bed marker is radiopaque, continuous, multi-plane, inert, non-palpable, non-absorbable, maintains position along the entirety of the length of each 


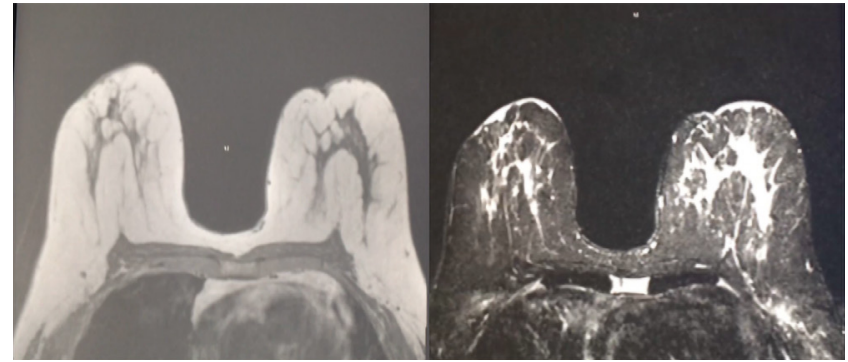

Figure 8 Six-week post-operative MRI, after left breast lumpectomy and right breast excisional biopsy (both sites marked the filament marker). MRI, magnetic resonance imaging.

margin (regardless of where that margin may be after reconstruction), does not create artifact, and takes up negligible to no measurable space so as to not impede a full thickness closure with or without additional oncoplastic techniques.

In the absence of an accurate tumor bed marker, radiation oncologists are delivering an adjusted radiation volume to compensate for uncertainty in size, location, and/or shape of the actual tumor bed $(9,26,28,39,40)$. The delivered volume is also currently used as a surrogate for attempting to measure or assess the accuracy of XRT $(17,27,28)$. Traditionally available tumor bed markers entail single point identification of margin(s), limiting accurate definition of the actual true tumor bed margins. The filament marker allots for complete tumor bed identification on radiographic follow-up and CT XRT planning via an inherently simple and intuitive method to identify its true location and extent. Its adaptable nature allows marking in any type of breast surgical procedure without inhibiting or limiting procedural technique. The applicability of the filament marker may be particularly timely given the recent B39 findings demonstrating the absolute difference in 10 -yr rate of IBTR was $<1 \%$ (4.8\% PBI vs. $4.1 \%$ WBI); the risk of an RFI event was statistically significantly higher for PBI compared to WBI, but the absolute difference in 10-yr RFI rate was also small (8.1\% PBI vs. 6.6\% WBI); and DDFI, OS, and DFS were not statistically different for PBI vs. WBI (41). The filament marker is a definitive, non-palpable, non-absorbable, radiopaque continuous multiplane marker outlining the entire length of each tumor border margin, resulting in easy identification of the true tumor bed, regardless of where the respective tumor bed margins may be after surgery. Radiation oncologists have a definitive, visible, accurate target, for planning and delivery of XRT (Figure 9). The decades old method of

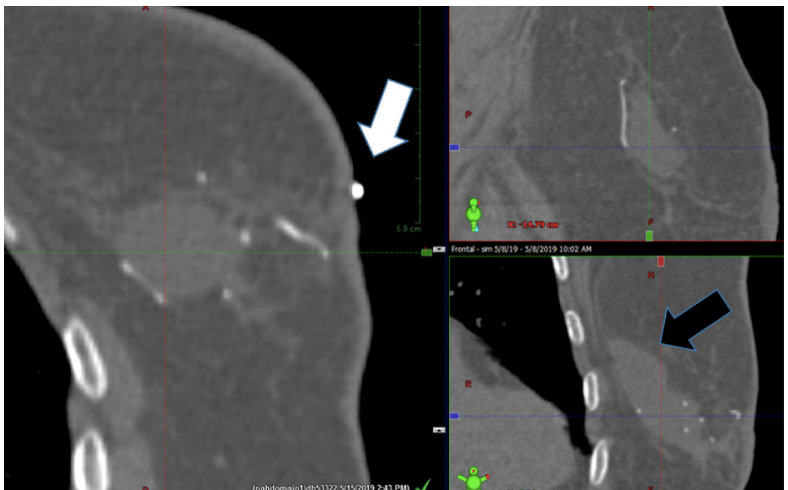

Figure 9 Axial, sagittal, and coronal radiation therapy planning with the filament marker. CT images, 5 weeks after left breast lumpectomy. A wire (white arrow) is seen along the skin incision. Note that the seroma shadow (black arrow) overestimates the actual tumor bed delineated by the marker in the sagittal projection. CT, computed tomography.

relying on inaccurate surrogates, resulting in potential increased volume of therapy to compensate for uncertainty, is eliminated. Utilization of the filament marker thus eliminates the need to rely on surrogates, decreases extra volume for uncertainty, and allows for precise/accurate XRT administration to the boost volume. Radiologists are able to clearly assess the actual tumor bed site on subsequent follow-up imaging without artifact, and with negligible to no risk of (marker) displacement.

\section{Conclusions}

The filament marker is a non-palpable adaptable radiographically opaque multi-plane tissue marker conformable to any breast surgical procedure. Placement is a simple technique requiring only a needle driver and scissors-readily available on every operating room table. Utilization affords radiologists the advantage of clear identification on subsequent follow-up imaging and optimal targeting for radiation oncologists in XRT planning, with resultant more precise and accurate delivery of XRT. The clearly defined continuous multi-plane marker placed along the tumor bed margins better delineates the actual tumor bed margins, inherently taking the guess work out of XRT planning and radiographic follow-up.

\section{Acknowledgments}

None. 


\section{Footnote}

Conflicts of Interest: Dr. Mitchell, Dr. Lee, Dr. DuPree, Dr. Beyer, Dr. Grobmyer, Dr. Gass, Dr. Boolbol, and Dr. Storm-Dickerson are participants in Videra Surgicals Clinical Advisory Board and received no monetary support or payment for this work. M Ulissey has no conflicts of interest to declare.

Ethical Statement: The authors are accountable for all aspects of the work in ensuring that questions related to the accuracy or integrity of any part of the work are appropriately investigated and resolved. Patients gave informed consent for utilization of their de-identified images and medical information for the purposes of research and education. No ethics approval was required for the purposes of this reporting of findings.

\section{References}

1. Prendergast B, Indelicato DJ, Grobmyer SR, et al. The dynamic tumor bed: volumetric changes in the lumpectomy cavity during breast conserving therapy. Int J Radiat Oncol Biol Phys 2009;74:695-701.

2. Hlavka A, Vanasek J, Odrazka K, et al. Tumor bed radiotherapy in women following breast conserving surgery for breast cancer-safety margin with/without image guidance. Oncol Lett 2018;15:6009-14.

3. Chung MJ, Lee GJ, Suh YJ, et al. Setup Error and Effectiveness of Weekly Image-Guided Radiation Therapy of TomoDirect for Early Breast Cancer. Cancer Res Treat 2015;47:774-80.

4. Snider JW, Mutaf Y, Nichols E, et al. Dosimetric improvements with a novel breast stereotactic radiotherapy device for delivery of preoperative partial-breast irradiation. Oncology 2017;92:21-30.

5. Rault E, Lacornerie T, Dang HP, et al. Accelerated partial breast irradiation using robotic radiotherapy: a dosimetric comparison with tomotherapy and three-dimensional conformal radiotherapy. Radiat Oncol 2016;11:29.

6. van der Salm A, Murrer L, Steenbakkers I, et al. Actual target coverage after setup verification using surgical clips compared with external skin markers in postoperative breast cancer radiation therapy. Pract Radiat Oncol 2017;7:e369-76.

7. Zhang A, Li J, Qiu H, et al. Comparison of rigid and deformable registration through the respiratory phases of four-dimensional computed tomography image data sets for radiotherapy after breast-conserving surgery. Medicine (Baltimore) 2017;96:e9143.

8. Zhang A, Li J, Wang W, et al. A comparison study between gross tumor volumes defined by preoperative magnetic resonance imaging, postoperative specimens, and tumor bed for radiotherapy after breast-conserving surgery. Medicine (Baltimore) 2017;96:e5839.

9. Yang TJ, Tao R, Elkhuizen PH, et al. Tumor bed delineation for external beam accelerated partial breast irradiation: a systematic review. Radiother Oncol 2013;108:181-9.

10. Verhoeven K, Peeters S, Erven K, et al. Boost delineation in breast radiation therapy: Isotropic versus anisotropic margin expansion. Pract Radiat Oncol 2016;6:e243-8.

11. Thomas K, Rahimi A, Spangler A, et al. Radiation practice patterns among United States radiation oncologists for postmastectomy breast reconstruction and oncoplastic breast reduction. Pract Radiat Oncol 2014;4:466-71.

12. de Freitas TB, Lopes de Barros Lima KM, de Andrade Carvalho $\mathrm{H}$, et al. What a difference a clip makes! Analysis of boost volume definition in radiation therapy for conservative breast surgery. Eur J Surg Oncol 2018;44:1312-7.

13. Clough KB, Kaufman GJ, Nos C, et al. Improving breast cancer surgery: a classification and quadrant per quadrant atlas for oncoplastic surgery. Ann Surg Oncol 2010;17:1375-91.

14. Fitoussi A, Berry MG, Couturaud B, et al. Oncoplastic and reconstructive surgery for breast cancer. The institute Curie experience. Berlin: Springer, 2009.

15. El-Tamer MB. Principles and techniques in oncoplastic breast cancer surgery. Singapore: World Scientific, 2013.

16. Mitchell SD. A step-by-step oncoplastic breast conservation surgical atlas of reproducible dissection techniques and anatomically ideal incision placement. Breast Cancer Res Treat 2017;165:505-16.

17. Wu SD, Fan Y, Kong J, et al. Single incision for quadrantectomy and laparoscopic axillary lymph node dissection in the treatment of early breast cancer: initial experience of 5 cases. J Laparoendosc Adv Surg Tech A 2014;24:791-4.

18. Alço G, Igdem S, Okkan S, et al. Replacement of the tumor bed following oncoplastic breast-conserving surgery with immediate latissimus dorsi mini-flap. Mol Clin Oncol 2016;5:365-71.

19. Sung Sy, Lee JH, Lee JH, et al. Displacement of Surgical Clips during Postoperative Radiotherapy in Breast Cancer Patients Who Received Breast-Conserving Surgery. J 
Breast Cancer 2016;19:417-22.

20. Kirwan CC, Al Sarakbi W, Loncaster J, et al. Tumour bed clip localization for targeted breast radiotherapy: compliance is proportional to trial-related research activity: tumour bed clip localization in breast radiotherapy. Eur J Surg Oncol 2014;40:158-62.

21. Kirby AN, Jena R, Harris EJ, et al. Tumour bed delineation for partial breast/breast boost radiotherapy: what is the optimal number of implanted markers? Radiother Oncol 2013;106:231-5.

22. Guo B, Li J, Wang W, et al. Interobserver variability in the delineation of the tumour bed using seroma and surgical clips based on 4DCT scan for external-beam partial breast irradiation. Radiat Oncol 2015;10:66.

23. Ebner F, de Gregorio N, Rempen A, et al. To clip or not to clip the breast tumor bed? A retrospective look at the geographic miss index and normal tissue index of 110 patients with breast cancer. J Turk Ger Gynecol Assoc 2017;18:67-71.

24. Wang W, Li J, Xing J, et al. Analysis of the variability among radiation oncologists in delineation of the postsurgical tumor bed based on 4D-CT. Oncotarget 2016;7:70516-23.

25. Wang S, Li J, Wang W, et al. A study on the displacements of the clips in surgical cavity for external-beam partial breast irradiation after breast-conserving surgery based on 4DCT. J Radiat Res 2012;53:433-8.

26. Kirova YM, Castro Pena P, Hijal T, et al. Improving the definition of tumor bed boost with the use of surgical clips and image registration in breast cancer patients. Int J Radiat Oncol Biol Phys 2010;78:1352-5.

27. Furet E, Peurien D, Fournier-Bidoz N, et al. Plastic surgery for breast conservation therapy: how to define the volume of the tumor bed for the boost? Eur J Surg Oncol 2014;40:830-4.

28. Ippolito E, Trodella L, Silipigni S, et al. Estimating the value of surgical clips for target volume delineation in external beam partial breast radiotherapy. Clin Oncol (R Coll Radiol) 2014;26:677-83.

29. Atrchian S, Sadeghi P, Cwajna W, et al. Improvement of consistency in delineating breast lumpectomy cavity using surgical clips. J Surg Res 2018;221:30-4.

30. Lee PY, Lin CY, Chen SW, et al. A topology-based method to mitigate the dosimetric uncertainty caused by the positional variation of the boost volume in breast conservative radiotherapy. Radiat Oncol 2017;12:55.

31. Penninkhof J, Quint S, Baaijens M, et al. Practical use of the extended no action level (eNAL) correction protocol for breast cancer patients with implanted surgical clips. Int
J Radiat Oncol Biol Phys 2012;82:1031-7.

32. Fassi A, Ivaldi GB, de Fatis PT, et al. Target position reproducibility in left-breast irradiation with deep inspiration breath-hold using multiple optical surface control points. J Appl Clin Med Phys 2018;19:35-43.

33. Trovo M, Polessel J, Biasutti C, et al. Fiducial markers for image-guided partial breast irradiation. Radiol Med 2013;118:1212-9.

34. Zhang Y, Mutter RW, Park SS, et al. Carbon Fiducial Image Guidance Increases the Accuracy of Lumpectomy Cavity Localization in Radiation Therapy for Breast Cancer. Pract Radiat Oncol 2019;9:e14-21.

35. Trombetta MG, Hasan S, Malay MB, et al. Treatment volume reduction using the biozorb device in ipsilateral breast recurrence treated with second conservation therapy. JSM Clin Case Rep 2017;5:1134.

36. Cross $\mathrm{M}$, Ross J, Jones $\mathrm{S}$, et al. Identifying the surgical cavity after oncoplastic breast surgery. ASCO 2014 Breast Cancer Symposium. Available online: http://focalrx.com/ wp-content/uploads/2014/01/FMRK-11-07_Rev-A_ Poster-Handout_ASCO-Breast-2014_PRINTABLE.pdf

37. Wiens N, Torp L, Wolff B, et al. Effect of BioZorb surgical marker placement on post-operative radiation boost target volume. J Radiat Oncol 2018;7:175-9.

38. Rashad R, Huber K, Chatterjee A. Cost-Effectiveness of the Biozorb Device for Radiation Planning in Oncoplastic Surgery. Cancer Clin Oncol 2018;7:23-32.

39. Hansen CJ, de Winton E, Guglani S. et al. Target localization for tumour bed radiotherapy in early breast cancer. J Med Imaging Radiat Oncol 2012;56:452-7.

40. Pirlamarla A, Ferro A, Yue NJ, et al. Optimization of Surgical clip placement for breast conservation therapy. Pract Radiat Oncol 2014;4:153-9.

41. Vicini FA, Cecchini RS, White JR, et al. Abstract GS404: Primary results of NSABP B-39/RTOG 0413 (NRG Oncology): A randomized phase III study of conventional whole breast irradiation (WBI) versus partial breast irradiation (PBI) for women with stage 0 , I, or II breast cancer. Cancer Res 2019. doi: 10.1158/1538-7445. SABCS18-GS4-04.

Cite this article as: Mitchell S, Lee H, DuPree BB, Beyer DC, Ulissey M, Grobmyer SR, Gass J, Boolbol S, Storm-Dickerson T. A novel, adaptable, radiographically opaque, multi-plane continuous filament marker for optimizing tissue identification, radiation planning, and radiographic follow-up. Gland Surg 2019;8(6):609-617. doi: 10.21037/gs.2019.10.03 